\title{
The Relationship between Endothelial Nitric Oxide Synthase with Dyslipidemia in Coronary Heart Disease
}

\author{
Eryati Darwin $^{1 *}$, Eka Fithra Elfi², Eva Decroli ${ }^{3}$, Dwitya Elvira ${ }^{3}$ \\ ${ }^{1}$ Department of Histology, Faculty of Medicine, Universitas Andalas, Padang, West Sumatera, Indonesia; ${ }^{2}$ Department of \\ Cardiology, Faculty of Medicine, Universitas Andalas, Padang, West Sumatera, Indonesia; ${ }^{3}$ Department of Internal Medicine, \\ Faculty of Medicine, Universitas Andalas, Padang, West Sumatera, Indonesia
}

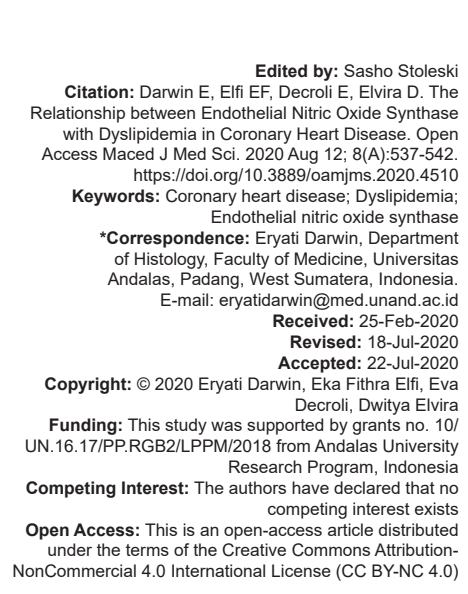

\section{Abstract}

BACKGROUND: Dyslipidemia is a medical condition that refers to an abnormal of blood lipids level, most common is hyperlipidemia. Elevation of blood lipid such as cholesterol, triglycerides, low-density lipoprotein (LDL) cholesterol, or a low high-density lipoprotein (HDL) cholesterol level induces disturbance of endothelial function. Endothelia dysfunction in dyslipidemia is in large part due to a reduced bioavailability of nitric oxide (NO) which is synthesized by endothelial NO synthase (eNOS) which will trigger the development of atherosclerosis, and increases the risk of coronary heart disease (CHD).

AIM: This study aims to determine the relationship of lipid profiles of dyslipidemia with eNOS levels in CHD patients.

MATERIALS AND METHODS: This study design was a cross-sectional study. The sample in this study was 26 patients which diagnosed as CHD of outpatients in the department of cardiology of a regional general hospital and fulfilled the inclusion and exclusion criteria. Fifteen healthy individuals who come for the general check-up were included as a control group. Blood samples were collected for measurements of eNOS levels and lipid profiles that carried out according to laboratory standards. Statistical analyses were conducted using analysis of variance and Shapiro-Wilk test.

RESULTS: The results of this study showed that eNOS levels in the group of CHD patients $(24.174 \pm 7.136 \mathrm{ng} / \mathrm{ml})$ were significantly lower $(p<0.05)$ than those in the control group $(72.092 \pm 30.065 \mathrm{ng} / \mathrm{ml})$. The mean of triglycerides and HDL levels has a weak correlation with the level of eNOS $(p>0.05)$. The mean of LDL level has moderately positive correlation with eNOS, while cholesterol has a moderate negative correlation with the level of eNOS ( $p>$ 0.05).

CONCLUSION: This study concluded that high cholesterol levels and low HDL levels in dyslipidemia condition were correlated with eNOS, which has a protective function in CHD.

\section{Introduction}

Coronary heart disease (CHD) is a result of coronary artery disease (CAD), leading cause of death and disability worldwide. The prevalence of $\mathrm{CHD}$ is expected to increase in the coming years [1]. $\mathrm{CHD}$ refers to a narrowing of the coronary arteries, the blood vessels that supply oxygen and blood to the heart. This is usually caused by atherosclerosis which is a buildup of plaque inside the artery wall that can start in childhood and may be well advanced by middle age [2], [3]. The plaque first grows within the walls of the coronary arteries and continues to develop until the blood flow to the heart's muscle decreases. This can be chronic, by the narrowing of the coronary artery and reduce of the blood supply to the heart muscle, which may lead to prevent the heart muscle from receiving enough oxygen, referred as myocardial ischemia, or it can be acute, due to a sudden rupture of a plaque and formation of a thrombus or blood clot, that could lead to heart attacks [4], [5].
CHD is caused by the interplay of genetic and environmental factors, the effects of which are mainly mediated through cardiovascular risk factors. There are many risk factors for CHD that can be controlled such as high blood pressure, high blood cholesterol levels, as well as the effects of related factors such as blood triglyceride, low-density lipoprotein (LDL) cholesterol level, and high-density lipoprotein (HDL) cholesterol levels, diabetes mellitus, overweight or obesity, lack of physical activity, smoking, unhealthy diet, and psychosocial factors. Those cannot be controlled such as age, sex, family history, and race. The multiple interrelated factors seem responsible for occurrence of CHD [6], [7].

Atherosclerosis is a lipoprotein-driven disease that leads to plaque develops in the arterial wall characterized by accumulation of lipids, fibrous, and inflammatory molecules. Lipoproteins are complex plasma particles containing a core of cholesterol esters and triglycerides surrounded by free cholesterol, phospholipids, and apolipoproteins. The size, structure, and apolipoprotein content of the lipoproteins, 
namely, chylomicrons, very LDL, intermediate-density lipoprotein, LDL, HDL, and lipoprotein a, crystallize into individualized atherosclerotic risk profiles for the specific lipoprotein. Medical condition refers to an abnormal level of blood lipids levels known as dyslipidemia. The term describes a wide range of conditions, the most common forms of dyslipidemia involve: High levels of LDL, low levels HDL, high levels of triglycerides, and high cholesterol, which refers to high LDL and triglyceride levels [6], [8].

High levels of triglycerides and LDL cholesterol (LDL-C) damage vascular endothelial cells and their functions. Efflux of LDL cholesterol to the subendothelial space, which can then be modified and oxidized by various agents promotes monocyte adhesion and migration to the subendothelial space. Monocytes differentiate to macrophages and become foam cells that have pro-inflammatory functions, including the release of cytokines such as interleukins and tumor necrosis factor. Endothelial dysfunction is mediated through several diverse mechanisms that include increased oxidative stress and pro-inflammatory responses [9], [10]. Non-HDL cholesterol contributes to impairment of nitric oxide (NO) bioavailability due to increased production of reactive oxygen species and inhibition of endothelial NO synthase (eNOS) activity that leads to endothelial dysfunction. Disruption of endothelial function is considered a key event in the development and progression of atherosclerosis [11].

eNOS is a constitutively expressed enzyme that modulates endothelial functions, expressed mainly in the endothelium of large arteries which decrease in smaller arteries and disappear in capillary. eNOS is primarily responsible for the generation of $\mathrm{NO}$ in the vascular endothelium. NO produced by eNOS in the vascular endothelium plays crucial roles in regulating vascular tone, cellular proliferation, leukocyte adhesion, and platelet aggregation. Therefore, a functional eNOS is essential for a healthy vascular system [11], [12]. Elevated levels of LDL decrease the bioavailability of endothelium-derived NO, reduce $\mathrm{NO}$ production in part by increasing the interaction between caveolin-1 and eNOS, and downregulate endothelial eNOS [10].

The purpose of this study was to determine whether there is an association between dyslipidemia (triglyceride, HDL cholesterol [HDL-C], LDL-C, and total cholesterol) with eNOS levels in CHD patients.

\section{Materials and Methods}

The design of this study was cross-sectional study. Participants were enrolled in this study, which were the outpatient in the department of cardiology of a regional general hospital in Padang.

\section{Samples}

Subjects in this study were 26 patients in the age of $35-55$ years old, diagnosed with CHD by the cardiologist clinically with electrocardiogram and laboratory confirmation. All subjects fulfill the inclusion and exclusion criteria, such as a history of malignancies, infectious diseases, and other autoimmune diseases. Fifteen healthy individuals who come for the general check-up were included as a control group. Venous blood samples were collected after a $12 \mathrm{~h}$ fast by certified technicians using standardized venipuncture procedure from the patients cubital veins for the measurement of lipid profile and eNOS levels.

\section{Examination of serum lipid profile}

Serum was measured for lipids panel by an enzymatic colorimetric method using reagents of chemistry products on automated clinical chemistry analyzers. Based on laboratory standard, dyslipidemia was confirmed when the levels of triglyceride were lower than $150 \mathrm{mg} / \mathrm{dl}$, LDL below $100 \mathrm{mg} / \mathrm{dl}$, HDL above $50 \mathrm{mg} / \mathrm{dl}$, and total cholesterol lower than $200 \mathrm{mg} / \mathrm{dl}$.

Blood were collected into a glass tube, placed upright, and allowed for $45 \mathrm{~min}$ at room temperature and then centrifuge the blood samples at 1500× $g$ for $30 \mathrm{~min}$ at $4^{\circ} \mathrm{C}$. Sample was analyzed using Abbott Architect instrument, enzymatically and simultaneously using reagents from the same manufacturer. Place a $100 \mathrm{ul}$ aliquot of sample into the sample cups on the instrument carousel using a transfer pipet. Arrange the samples on the carousel in the order in which they are to be analyzed, as determined from the download file. The measurements were programmed by downloading the specimen ID numbers and test codes for a lipid panel includes total cholesterol, measures all the cholesterol in all the lipoprotein particles; HDL-C, measures the cholesterol in HDL particles; LDL-C, calculates or measures the cholesterol in LDL particles; and triglycerides, measures all the triglycerides in all the lipoprotein particles total cholesterol, triglyceride, HDL-C, and direct HDL-C, as appropriate in machine readable form. The results are printed on the Architect printout and also sent in real time to computer text files on a dedicated computer.

\section{Measurement of eNOS levels}

The level of eNOS was measured using monoclonal antibody against eNOS for ELISA methods. All measurements were following the manufacturer's protocol, carried out in the Biomedical Laboratory of the Medical Faculty, Andalas University. Human eNOS ELISA kit from Bioassay Technology Laboratory (Abcam E0908 Hu) is an in vitro enzyme-linked immunosorbent assay for the measurement of human pan-eNOS and total eNOS. The eNOS antibody has been coated onto a 96-well plate. Blood samples were centrifuged for 20 
min at $1000 \times \mathrm{g}$ at $4^{\circ} \mathrm{C}$, and then, $40 \mathrm{ul}$ serum of blood samples were pipetted into the wells and eNOS present in a sample is bound to the wells by the immobilized antibody. The wells were washed and $10 \mathrm{ul}$ mouse anti-eNOS antibody is used to detect pan eNOS. After washing away unbound antibody, 50 ul HRP-conjugated anti-mouse lgG is pipetted to the wells. The wells were again washed, a 90 ul TMB substrate solution was added to the wells and color develops in proportion to the amount of eNOS or eNOS bound. The stop solution changes the color from blue to yellow, and the intensity of the color is measured at $450 \mathrm{~nm}$.

\section{Ethical clearance}

This study involved human subjects so that it requires a statement of informed consent. All patients and healthy controls in the study provided written informed consent. The study protocol and all procedures were approved by the Institutional Review Board of Faculty of Medicine, Andalas University no.708/KEP/ $\mathrm{FK} / 2019$, and comply with the Helsinki Declaration of 1975 , as revised in 2000 .

\section{Statistical analysis}

The data analysis was done in line with study objectives. Values were expressed as mean \pm standard deviation, univariate and multivariate linear regression analyses were performed to evaluate the association of variables. Normally distributed variables were analyzed using one-way analysis of variance. Nonnormally distributed variables were analyzed using Mann-Whitney or Kruskal-Wallis tests. The Student's t-test was used for parametric data to compare the means of quantitative variables between two groups. A Chi-square test was used to compare frequencies across groups. Spearman's correlation was used for non-normally distributed variables. $p=0.05$ was considered to indicate a statistically significant difference.

\section{Results}

Lipid profiles in the CHD patients, as shown in Table 1, show that the average triglyceride levels were higher than normal levels (below $200 \mathrm{mg} / \mathrm{dl}$ ), LDL-C levels were higher than normal levels (below $100 \mathrm{mg} / \mathrm{dl}$ ), total cholesterol levels were higher than normal levels (150 mg/dl), while HDL-C levels were lower than normal levels (above $50 \mathrm{mg} / \mathrm{dl}$ ).

The results of eNOS levels measurement in our study showed that eNOS levels in CHD patients $(24.174 \pm 7.136)$ were significantly lower than controls group $(72.092 \pm 30.065)(p<0.05)($ Table 2$)$.
Table 1: Triglyceride, LDL cholesterol, HDL cholesterol, and total cholesterol levels in patients with $\mathrm{CHD}(n=26)$ in compare to normal standard levels

\begin{tabular}{llll}
\hline No. & Lipid profile & CHD $(\mathrm{mg} / \mathrm{dl})$ & Normal $(\mathrm{mg} / \mathrm{dl})$ \\
\hline 1 & Triglycerides & $211.50 \pm 55.53$ & $<200$ \\
2 & LDL & $136.76 \pm 29.39$ & $<100$ \\
3 & HDL & $39.08 \pm 10.19$ & $>50$ \\
4 & Cholesterol & $244.03 \pm 67.99$ & $<150$ \\
\hline
\end{tabular}

Simple linear regression analyses examining the relationship between eNOS with lipid profiles of dyslipidemia in CHD patients are shown in Figure 1.

Table 2: Average of eNOS levels $(\mathrm{ng} / \mathrm{ml})$ in the coronary heart disease patients and control group

\begin{tabular}{lllll}
\hline No. & Groups & $\mathrm{n}$ & Mean \pm SD & -value \\
\hline 1 & CHD & 26 & $24.174 \pm 7.136$ & $<0.05$ \\
2 & Control & 15 & $72.092 \pm 30.065$ & \\
\hline
\end{tabular}

The level of triglycerides has a weak correlation with the level of eNOS ( $p>0.05$ ) (Figure 1a). High level of LDL-C seems to contribute to the levels of eNOS, although was not significant $(p>0.05)$ (Figure $1 b)$. The results show that low level of HDL-C may decrease the level of eNOS, although was not significant different $(p>0.05)$ (Figure 1c). This result shows that high of total cholesterol levels are associated with significantly lower level of eNOS $(p<0.05)$ (Figure 1d).

\section{Discussion}

\section{Examination of serum lipid profile}

In this study, the number of CHD patients in men $(57.4 \%)$ was more than women $(42.3 \%)$, and in healthy individuals as a control group, men were $60 \%$ and women were $40 \%$. Both groups were in the age range of $35-55$ years. Men in the aged 35-54 years have a higher prevalence of cardiovascular disease than women of the similar age, but the gap tends to be narrower in recent years, with the prevalence decreased in men and increased in women [13]. Cardiovascular disease develops later in women than in men due to exposure of endogenous estrogens during the fertile period. Estrogens have a regulating effect on lipids metabolisms, inflammatory markers, coagulant system, and vascular tones [14]

The elevation of total cholesterol, triglyceride, or both, or a low HDL-C level called as dyslipidemia contributes to the development of atherosclerosis. High LDL cholesterol, low HDL cholesterol, high blood pressure, family history, diabetes mellitus, smoking, being postmenopausal for women and being older than 45 , and obesity for men are traditional risk factors of CHD [7]. Dyslipidemia is a major risk factor for CHD. Cholesterol level in blood plasma is determined by genetic and by consumption of high fat and cholesterol in the diet. In individuals at the CHD high risk, elevated LDL-C is considered to be the major cause of atherosclerosis and CHD. Therefore, the reduction 


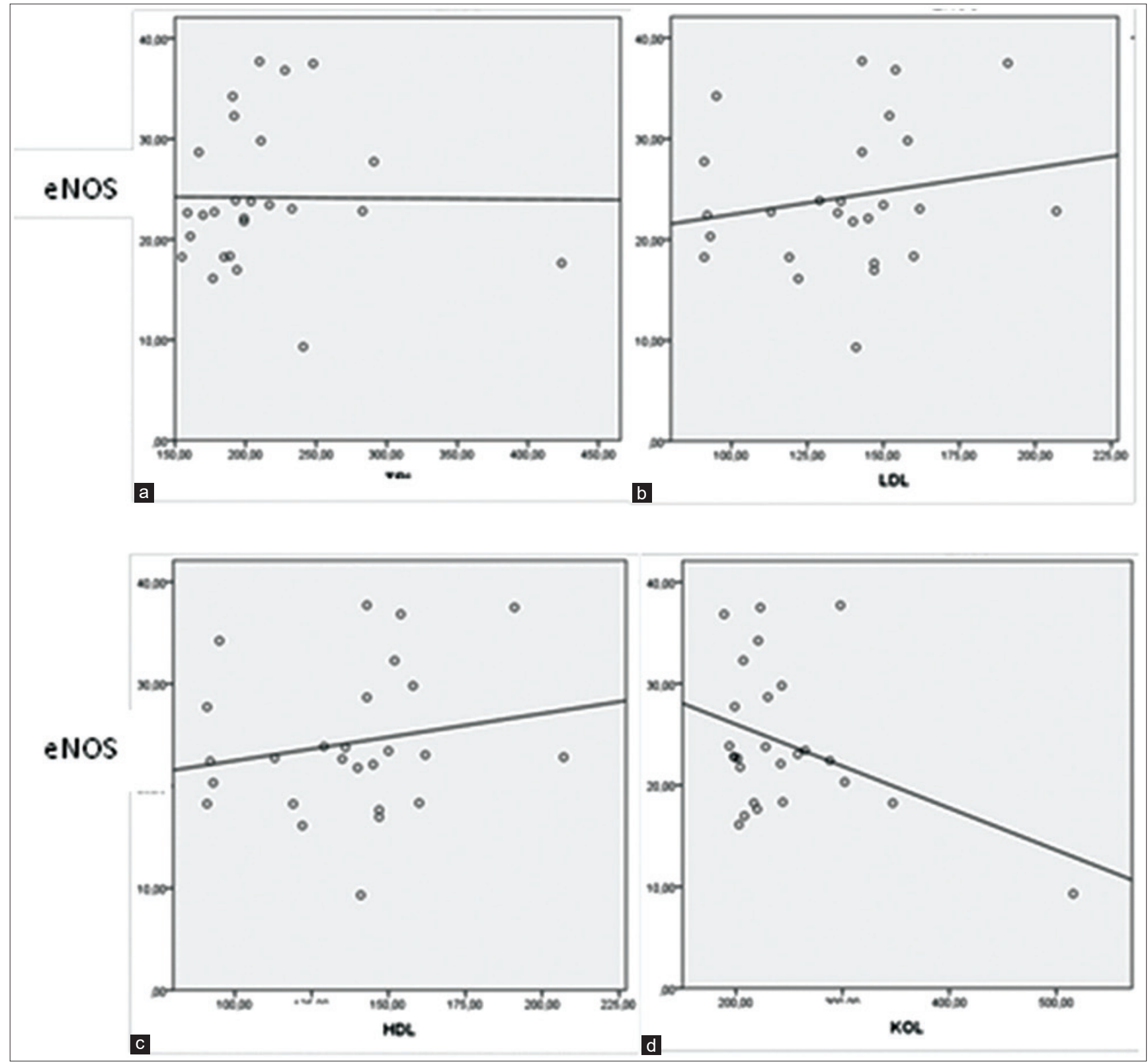

Figure 1: Correlation between thelial nitric oxide synthase with (a) triglyceride, (b) low-density lipoprotein, (c) high-density lipoprotein, (d) cholesterol

of LDL-C in these individuals may reduce the risk for CHD.

Other factors such as obesity and physical inactivity contribute to an elevation of cholesterol. The LDL-C is the predominant cholesterol-carrying lipoprotein and is considered to be the main atherogenic lipoprotein, in which LDL-C levels are closely correlated with CHD risk. Therefore, the high LDL-C levels than normal standard levels are a risk factor for CHD. In contrast, a high HDL-C level is considered to be protective against $\mathrm{CHD}$. Elevated triglyceride levels also are associated with increased CHD risk [6], [15]. A study of Orozco et al. [16] found that HDL-C total/HDL-C and triglycerides/HDL-C ratio were associated with a higher population attributable risk for cardiovascular disease.

\section{Measurement of eNOS levels}

High level of lipids in the blood is physical and biochemical injury to the endothelium of the coronary artery, leads to oxidative stress, eNOS uncoupling, and endothelial dysfunction. Vascular oxidative stress can be reduce and disrupt eNOS functionally. Low level of eNOS affects to NO production and function or bioavailability, resulting in increased vascular contractions to vasoconstrictors. Therefore, decrease of endothelial NO function or increase degradation of $\mathrm{NO}$ is associated with cardiovascular disorders including atherosclerosis as the base for the occurrence of CHD. The important function of eNOS is to keep blood vessels dilated, control blood pressure, vasoprotective, and anti-atherosclerotic effects. Although there is no evidence that eNOS is a "disease gene," many cardiovascular risk factors lead to induce 
oxidative stress production and cause endothelial dysfunction [17].

Experimental and clinical studies provide evidence that the dysfunctional eNOS/NO pathway and defects of endothelial NO function are associated with major cardiovascular risk factors such as hyperlipidemia, also have a predictive value for atherosclerotic disease progression, and as an early marker for various cardiovascular disorders [12]. The high levels of triglyceride in the group of $\mathrm{CHD}$ patients were not contribute to the level of eNOS. The change of HDL-C and HDL-C levels is well-established independent risk factors for cardiovascular disease, while triglycerides remain controversial. Circulating triglycerides level has been studied as a risk factor for cardiovascular events. Clinical trial has been done by Kajikawa et al. [18] suggested that triglycerides are an independent predictor of endothelial function. Lowering circulating triglyceride levels may improve endothelial function, leading to a decrease in cardiovascular events.

The LDL-C levels have moderately positive correlation with eNOS (Figure 1b). High LDL-C that impairs the capacity of NO production is an early step in atherogenesis. A mechanism of endothelial toxicity of LDL-C that alters the activity of the endothelial isoform of NO synthase (eNOS) involves the transcriptional activation of the gene encoding caveolin-1, acts as a negative regulator of eNOS. Treatment with the inhibitors of cholesterol synthesis abrogates caveolin-1 upregulation and restores eNOS activity in hypercholesterolemic mice [19].

The level of HDL has a weak correlation with the level of eNOS (Figure 1c). High-density lipoprotein cholesterol has pleiotropic effects including antioxidant, anti-apoptotic, anti-inflammatory, anti-thrombotic, or anti-proteolytic properties that account for endothelial protective action. HDL-C inhibits oxidation by the decrease in ROS production and has been shown to inhibit apoptosis of endothelial cells induced by different stimuli such as oxLDL and TNF- $\alpha$. HDL isolated from patients with CAD was shown to have a reduced potential to activate eNOS [20].

The level of total cholesterol has a moderately negative correlation with eNOS level (Figure 1d). Cholesterol is recognized as a major cause of macrovascular disease. The mechanism by which serum cholesterol elevations cause cardiovascular disease is not completely understood. Impaired endothelium-dependent vasodilation associated with elevated cholesterol levels could represent one such mechanism. Vascular endothelial dysfunction related to reduce of NO availability which promotes mechanism of arteriosclerosis. There are nonpharmacological and pharmacological therapies to repair vascular endothelial. These therapies are intended to increase eNOS activity and NO release, inhibit NO degradation [21].
Dyslipidemia, which characterized by elevated of triglycerides, total cholesterol, and LDL-C levels, or levels of HDL-C, is an important risk factor for $\mathrm{CHD}$. The change of this lipid levels may trigger the production of oxidative stress that related to deregulation of eNOS enzymatic activity and inactivation of NO. eNOS that plays a role in vascular protection appears to play an important role in endothelial dysfunction due to buildup of lipid that induces atherosclerosis in CHD.

Our study was conducted on small number of samples which was one of the main limitations that necessitate careful interpretation of results. Considering to the other risk factors for CHD, further studies on each risk factor would certainly be more conclusive. However, our data confirm the importance of eNOS role in dyslipidemia as a risk factor of CHD. $\mathrm{CHD}$ is an atherosclerotic disease as the result of diverse metabolic and immunologic mechanisms by the formation of fatty acid plaque. The change of lipids level in dyslipidemia, including low levels of HDL, high levels of LDL, triglycerides, and cholesterol, may trigger the production of oxidative stress that related to deregulation of eNOS enzymatic activity and inactivation of NO. NO is a signaling molecule that plays a key role in the pathogenesis of inflammation and cell activation that eventually leads to endothelial dysfunction. Endothelial dysfunction plays a key role in the induction and progression of atherosclerosis and consequently of CHD. Our result confirmed that eNOS was associated with the elevated of low-density lipoprotein (LDL) cholesterol and the triglyceride concentrations, and a decrease in high-density lipoprotein (HDL) cholesterol concentration in the blood.

\section{CONCLUSION}

This study concluded that high cholesterol levels and low HDL levels in dyslipidemia condition were correlated with eNOS, which has a protective function in CHD.

\section{References}

1. Sayols BS, Lluís GC, Lucas G, Elosua R. Pathogenesis of coronary artery disease: Focus on genetic risk factors and identification of genetic variants. Appl Clin Genet. 2014;7:15-32. https://doi.org/10.2147/tacg.s35301 PMid:24520200

2. World Health Organization. Global Atlas on Cardiovascular Disease Prevention and Control. Geneva: World Health Organization; 2011.

3. Sanchis GF, Perez QC, Leischik R, Lucia A. Epidemiology of coronary heart disease and acute coronary syndrome. Ann Transl 
Med. 2016;4(13):256. https://doi.org/10.21037/atm.2016.06.33 PMid:27500157

4. Marzilli M, Merz NB, Boden WE, Bonow RO, Capozza PG Chilian WM, et al. Obstructive coronary atherosclerosis and ischemic heart disease: An elusive link! J Am Coll Cardiol. 2012;60(11):951-6. https://doi.org/10.1016/j.jacc.2012.02.082 PMid:22954239

5. Sanz J, Moreno PR, Fuster V. The year in atherothrombosis. J Am Coll Cardiol. 2013;62(13):1131-43. https://doi.org/10.1016/j. jacc.2013.06.045

PMid:23916939

6. Leon A, Bronas UG. Dyslipidemia and risk of coronary heart disease: Role of lifestyle approaches for its management. Am J Lifestyle Med. 2009;3(4);257-73. https://doi.org/10.1177/1559827609334518

7. Shaima C, Moorthi PV, Shaheen NK. Cardiovascular diseases: Traditional and non-traditional risk factors. J Med Allied Sci. 2016;6(2):46-51. https://doi.org/10.5455/jmas.228597

8. Hajar R. Risk factors for coronary artery disease: Historical perspectives. Heart Views. 2017;18(3):109-14. https://doi. org/10.4103/heartviews.heartviews_106_17

PMid:29184622

9. Bentzon JF, Otsuka F, Virmani R, Falk E. Mechanisms of plaque formation and rupture. Circ Res. 2014;114(12):1852-66. https:// doi.org/10.1161/circresaha.114.302721

PMid:24902970

10. Kim JA, Montagnani M, Chandrasekran S, Michael J, Quon MJ Role of lipotoxicity in endothelial dysfunction. Heart Fail Clin. 2012;8(4):589-607. https://doi.org/10.1016/j.hfc.2012.06.012 PMid:22999242

11. Heiss C, Rodriguez MA, Kelm M. Central role of eNOS in the maintenance of endothelial homeostasis. Antioxid Redox Signal. 2015;22(14):1230-42. https://doi.org/10.1089/ars.2014.6158 PMid:25330054

12. Yang Z, Ming XF. Recent advances in understanding endothelial dysfunction in atherosclerosis. Clin Med Res. 2006;4(1):53-65. PMid:16595793

13. Towfighi A, Zheng L, Ovbiagele B. Sex-specific trends in midlife coronary heart disease risk and prevalence. Arch Intern Med. 2009;169(19):1762-6. https://doi.org/10.1001/ archinternmed.2009.318

PMid:19858433

14. Maas $A H$, Appelman YE. Gender differences in coronary heart disease. Neth Heart J. 2010;18(12):598-602. https://doi. org/10.1007/s12471-010-0841-y

PMid:21301622

15. Abd AM, Goyfman M, Chaus A, Dabbous F, Tamura L, Sandfort V, et al. The correlation of dyslipidemia with the extent of coronary artery disease in the multiethnic study of atherosclerosis. $J$ Lipids. 2018;2018:1-9. https://doi.org/10.1155/2018/5607349

16. Orozco BD, Gil GV, Redon J, Martin MJ, Pallares CV, Navarro PJ, et al. Lipid profile, cardiovascular disease and mortality in a Mediterranean high-risk population: The ESCARVAL-RISK study. PLoS One. 2017;12(10):e0186196. https://doi.org/10.1371/journal.pone.0186196 PMid:29045483

17. Förstermann U, Sessa WC. Nitric oxide synthases: Regulation and function. Eur Heart J. 2012;33(7):829-37. https://doi. org/10.1093/eurheartj/ehr304

PMid:21890489

18. Kajikawa M, Maruhashi T, Matsumoto T, Iwamoto $\mathrm{Y}$, Iwamoto $\mathrm{A}$, Oda N, et al. Relationship between serum triglyceride levels and endothelial function in a large community-based study. Atherosclerosis. 2016;249:70-5. https://doi.org/10.1016/j. atherosclerosis.2016.03.035

PMid:27065244

19. Hermida N, Balligand JL. Low-density lipoprotein-cholesterolinduced endothelial dysfunction and oxidative stress: The role of statins. Antioxid Redox Signal. 2014;20(8):1216-37. https:// doi.org/10.1089/ars.2013.5537 PMid:23924077

20. Tran DA, Diallo D, Delbosc S, Varela PL, Dang QB, Lapergue B, et al. $\mathrm{HDL}$ and endothelial protection. $\mathrm{Br} \mathrm{J}$ Pharmacol. 2013;169(3):493-511. https://doi.org/10.1111/bph.12174 PMid:23488589

21. Yasunobu HY, Nagata D, Suzuki E, Nishimatsu H, Suzuki J, Nagai R. Diagnosis and treatment of endothelial dysfunction in cardiovascular disease. Int Heart J. 2010;51(1):1-6. https://doi. org/10.1536/ihj.51.1

PMid:20145343 